\title{
Role of omega 3-fatty acids and multivitamins in gestation
}

\section{Irene Hösli*, Rosanna Zanetti-Daellenbach, Wolfgang Holzgreve and Olav Lapaire}

University of Basel, Department of Obstetrics and Gynecology, Switzerland

\begin{abstract}
There is a rising interest in a balanced and varied women's diet not only preconceptionally but also during pregnancy and in the breastfeeding period in order to reduce fetal, neonatal, and maternal risks. A decreased intake of omega 3-fatty acids (FA) or deficiencies of micronutrients are a global health problem, not only in developing countries, where micronutrient-rich food and fortified aliments are often not available, and also in the industrialized world. In contrast to data about the daily periconceptionally intake of folate acid that has been shown to reduce substantially the occurrence and recurrence of neural tube defects (NTD), the benefit of other micronutrients is less determinant.

Deficiencies of omega 3-FA may be a contributing factor for severe complications in pregnancy and postpartum. A meta-analysis for the role of omega 3-FA in preeclampsia and maternal postpartum depression is less consistent, some meta-view's results differ substantially or are even contradictory to large observational studies. Further well-designed studies are warranted. A personal interview and counseling concerning the daily diet should be integrated in the preconceptional and in the antenatal care and an individual supplementation should be offered, if indicated.
\end{abstract}

Keywords: Dietary supplements; fatty acids; omega-3; pregnancy; vitamins.

\section{Role of omega 3-FA in gestation}

\section{Nutrition habits among women in industrialized countries}

Low pre-pregnancy body mass index (BMI) as well as low energy intake during pregnancy, such as seen in famine periods, is associated with adverse pregnancy outcome

\footnotetext{
${ }^{\star}$ Corresponding author:

PD Dr. I. Hösli

Universitäts-Frauenklinik Base

Spitalstrasse 21, CH-4031 Basel

Switzerland

Tel.: +41612659017

Fax: +41612659198

E-mail: ihoesli@uhbs.ch
}

like preterm delivery, intrauterine growth restriction (IUGR) and maternal delivery complications [13]. However, during the last 10-15 years, the BMI has increased to values over $30 \mathrm{~kg} / \mathrm{m}^{2}$ in $33.4 \%$ of women in the USA or in $9.2 \%$ of women in Switzerland, which has also influenced pregnancy outcome by increased rates of macrosomia, cesarean section, the rate of gestational diabetes, preeclampsia, preterm delivery and stillbirth [6]. In 2006, worldwide, more than a billion people are overweight $\left(\mathrm{BMI}>25 \mathrm{~kg} / \mathrm{m}^{2}\right)$, more than 300 million of them obese $\left(\mathrm{BMI}>30 \mathrm{~kg} / \mathrm{m}^{2}\right)$ compared to 800 million that are underweight $\left(\mathrm{BMI}<19 \mathrm{~kg} / \mathrm{m}^{2}\right)$ [27].

Fat intake worldwide has increased substantially, mainly consisting of the saturated and trans fatty acids, followed by a decline of polyunsaturated fatty acids (PUFA) and especially of omega $3-F A$ of about $80 \%$. Measurements of human milk concentrations of decosahexaenoic acid (DHA) showed a decrease by $50 \%$ in Canada and Australia [23]. At the same time, the intake of omega 6 FA increased and the dietary ratio of omega 6-3-FA changed from the earlier $2: 1$ to the current 10-16:1 [36]. One explanation for a decline in omega 3-FA rich diet amongst other factors - is an increasing concern from environmental pollutants like heavy metals that are known to accumulate in certain fish species. Fish that contain high levels of mercury, polychlorinated biphenyls (PCBs) or dioxin are mostly the long living and predatory fish types like tilefish, swordfish, shark and king mackerel. This has led to warnings from national organizations like the Food and Standard Agency (FSA) to reduce fish consumption, the richest source of omega 3-FA. After the release of this advice the fish consumption in the USA decreased continually for all fish groups. Unfortunately this included canned tuna fish, which contains small fish, and has far lower mercury levels. Although different national guidelines reinforced a weekly intake of two portions of fish from different sources, the trend for avoiding fish and, therefore, omega 3-FA continues [28].

\section{Demand of omega 3-FA in pregnancy and lactating period}

DHA concentration in maternal plasma increases early in pregnancy (approximately $40 \%$ in the first and second trimester) compared to pre-pregnancy levels. The DHA concentration subsequently depletes as the demand of the fetus increases in the third trimester [22]. The increase in maternal plasma cannot be explained by an increased intake, but is caused by mobilization from maternal stores built up before pregnancy. If the mother is breastfeeding after delivery, the maternal blood con- 
centration of DHA rapidly decreases by up to $20 \%$ under normal value and returns after weaning. If the baby is bottle-fed, the DHA concentration soon after delivery returns to the pre-pregnancy level.

Several influences of omega 3-FA on the pregnant women and the fetus have been assessed in observational studies analyzing maternal fish intake and in randomized controlled studies with the supplementation of fish oil (Table 1).

\section{Influence of omega 3-FA on preeclampsia}

Maternal blood pressure responds to fish oil supplementation in a dose dependent manner. The result of a metaregression analysis of randomized controlled trials (RCT) showed that the systolic blood pressure decreased by $2.1 \mathrm{mmHg}(95 \% \mathrm{Cl} 1.0-3.2)$ and the diastolic blood pressure by $1.5 \mathrm{mmHg}(95 \% \mathrm{Cl} 1.0-2.2)$ in a non pregnant population with a median dose of $3.7 \mathrm{~g} / \mathrm{d}$ fish oil mainly comprising eicosapentanoic acid (EPA)/DHA [16].

Preeclampsia is one of the most common serious complications in pregnancy, affecting $5-7 \%$ of pregnant women and is one of the leading causes for maternal morbidity and mortality. As preeclampsia is a state of increased oxidative stress and a reduced antioxidant response there are several theoretical rationales for the use of omega 3-FA as antioxidants:

1. Inhibiting the prostaglandin synthesis and exhibit antioxidant properties [29]

2. Decreasing platelet and leukocyte reactivity

3. Vasodilatation

Epidemiological observational studies confirmed that Inuit women with a high consumption of fish had a lower blood pressure at the end of pregnancy and a lower incidence of pregnancy induced hypertension [11]. Cord blood phospholipids fatty acid analyses confirmed higher levels of EPA, a fatty acid enriched in fish and sea mammals [17]. In a cross sectional study where erythrocyte omega 3- and 6-FA as biomarkers were measured, women with the lowest levels of omega 3-FA were 7.6 times more likely to have pregnancies complicated by preeclampsia as compared with women with the highest levels of omega 3-FA after adjusting for confounders $195 \%$ $\mathrm{Cl} 1.4-40.6)$. A $15 \%$ increase in the ratio of omega

Table 1 The deficit of omega 3-fatty acids has the potential to influence both maternal and fetal health during pregnancy.

\begin{tabular}{ll}
\hline Maternal health & Fetal development \\
\hline Preeclampsia, eclampsia & Preterm delivery \\
Gestational hypertension & Birth weight \\
Gestational diabetes & Prolongation of pregnancy \\
Postpartum depression & IUGR \\
& Fetal neurological and visual \\
& development \\
\hline
\end{tabular}

3-6-FA was associated with a $46 \%$ reduction in risk of preeclampsia (OR $=0.54 ; 95 \% \mathrm{Cl} 0.41-0.72)$ [42]. As omega 3-FA might have both antioxidative and pro-oxidative properties, depending on experimental conditions, dosage and background of the diet, Shoji et al. [35] documented in a double blind RCT that supplementation of $500 \mathrm{mg}$ DHA and $150 \mathrm{mg}$ EPA combined with Vitamin A, $E, D$ and $C$ in the required amount during pregnancy did not enhance oxidative DNA damage and lipid peroxidation in 23 pregnant women from 20 weeks of gestation to delivery. RCT on omega 3-FA and preeclampsia, however, failed to confirm a significant risk reduction.

Makrides et al. [26] summarized the data from 6 RCTs including 2783 women. The relative risk for developing preeclampsia after intake of marine fish oil or supplementation with any prostaglandin precursor showed in four trials with 1683 women and a singleton pregnancy, a trend to lower the risk for preeclampsia but no clear difference in the relative risk of preeclampsia between the two groups: RR 0.86 (95\% Cl 0.59-1.27). The studies differed in the amount of DHA intake between $133 \mathrm{mg} / \mathrm{d}$ and $3 \mathrm{~g} / \mathrm{d}$, the most commonly used dose was $2.7 \mathrm{~g} / \mathrm{d}$ PUFA (1.5 g EPA and $1 \mathrm{~g} \mathrm{DHA}$ ) corresponding to $300 \mathrm{~g}$ of cooked salmon daily. Supplementation started after 16 weeks of gestation. There was no difference in the percentage of bleeding complications before or after delivery between the supplemented and the control group. Thirteen percent had a blood loss over $500 \mathrm{~mL}$ in the fish oil group vs. $8 \%$ in the olive oil group. The only maternal side effects were belching and an unpleasant taste, RR 3.55 (95\% Cl 2.78-4.52). The authors concluded that there is not enough evidence to supplement women with fish oil or another prostaglandin precursor in order to reduce the risk for preeclampsia.

Another meta-analysis of 6 RCTs with 1278 low risk participants [39] did not find, in a subgroup of 328 women, evidence that supplementation with omega 3-FA/ DHA (1000 mg/d DHA vs. $200 \mathrm{mg} / \mathrm{d}$ DHA) influenced the rate of preeclampsia or eclampsia $(R R=0.73 ; 95 \% \mathrm{Cl}$ $0.22-2.37$ ). The trials differed in the beginning of supplementation as early as 15 weeks or as late as 30 weeks.

\section{Influence of omega 3-FA on postpartum depression}

Postpartum depression (PPD) affects between 5 and $20 \%$ of parturients. However, it is labeled as the most under-recognized, under-diagnosed, and under-treated obstetrical complication. PPD influences adversely the mother-child relationship, infant development and wellbeing.

Parturients provide an excellent opportunity to examine the relation between concentration of omega 3-FA and the prevalence of PPD because omega 3-FA are transferred from mother to child through breast milk. Hence new mothers are more likely to be depleted in omega 3-FA. DHA has been proposed in PPD as prophylaxis or treatment alone or adjunct to antidepressive 
therapy. The assumed physiologic mechanisms of omega 3-FA affecting postpartum depression are: [15]

1. Effects on serotonergic and dopaminergic neurotransmission

2. Alteration of protein phosphorylation and impact upon protein kinase

3. Immunological effects

4. Vagal mechanisms

5. Heightened dendritic arborization and synapse genesis

6. Regulation of gene expression

In observational studies, low levels of omega 3-FA measured in blood samples correlated with clinical depression, depressed mood and PPD. In general, these studies report a shift away from omega 3-FA towards omega 6-FA in depressed patients [37]. In four of seven double-blind RCTs performed in a general population, depression was significantly improved upon treatment with at least $1 \mathrm{~g} /$ day of EPA. It remained unclear whether omega 3-FA supplementation is effective independently of antidepressant treatment, or only for those with abnormally low concentrations of these PUFAs [37].

Tanskanen et al. [40] observed a significant relationship between infrequent fish consumption and depressive symptoms only among women but not in men. A multinational ecological study confirmed that both higher concentrations of DHA in mothers' milk ( $r=-0.84$, $\mathrm{P}<0.0001, \mathrm{n}=16$ countries) and greater seafood consumption ( $r=-0.81, P<0.0001, n=22$ countries) predict a lower prevalence rate of PPD in simple and logarithmic models, respectively [19]. The highest prevalence for PPD was $24.5 \%$ in South Africa and the lowest was $2.0 \%$ in Japan. A limitation was that confounding factors (young maternal age, low socioeconomic status, smoking, etc.) were not uniformly available.

The self report of 13,799 women with lower seafood intake at 32 weeks' gestation was associated with approximately doubling the risk of severe depression in pregnancy and postpartum [20]. One RCT investigated the effect of $0.2 \mathrm{~g} / \mathrm{d}$ DHA on PPD in 138 women. Llorente et al. [25] failed to observe an association, although DHA supplementation, given after delivery for four months, prevented the usual decline in plasma phospholipids DHA content of breastfeeding mothers. The limitations of this trial were the low dosage of DHA, treatment without EPA, and the fact that not all but only $6.7 \%$ of participants were diagnosed with clinical depression.

\section{International recommendations for omega 3-FA intake}

Evidence exists that the required intake of omega 3-FA does not meet requirements and that omega 3-FA does have a potential importance for pregnancy and the postpartum period. Although current available data from RCT are not sufficient to recommend general supplementa- tion, several organizations and scientific groups have made recommendations for fish consumption or supplementation for pregnant and lactating women:

- Food standard Agency (FSA)/Scientific Advisory Committee on Nutrition (SACN) 2003, UK: regular consumption of oily fish like fresh tuna, mackerel, sardines and trout, up to two portions/week, avoid shark, swordfish, marlin [2, 34].

- Food and drug administration (FDA)/Environmental protection agency (EPA) 2004: two average meals/ week of a variety of fish and shellfish that are lower in mercury (shrimps, canned tuna, salmon, pollock, catfish) and not more than one tuna steak (175 g)/week $[14,41]$.

- European community (EC) 2004: not more than $100 \mathrm{~g}$ of fish like swordfish, shark, marlin, and pickerel per week. Not more than two portions of tuna fish per week [12].

- Swiss health administration (BAG) 2006: oily fish consumption 1 to 2 times per week (280-300 g/week), fish with low mercury concentrations like trout, redfish, whitefish, sardines, white halibut. No consumption of swordfish, marlin, shark, high dioxin concentrations in Baltic herring and Baltic salmon [3].

- Perilip 2006: Maternal dietary intake of fatty fish or oils providing DHA of at least $200 \mathrm{mg} / \mathrm{d}$ up to $1 \mathrm{~g} / \mathrm{d}$ or $2.7 \mathrm{~g} / \mathrm{d}$ of PUFA [44].

It is recommended that only purified fish oil should be consumed. The quality of fish oil has to be tested for toxic contaminations like heavy metal, dioxins, PCBs. Hormones and any other contaminants should be absent.

\section{Micronutrients}

\section{Multiple micronutrient supplementations for women during pregnancy}

The combination of multiple micronutrients in a single pharmaceutical form has been suggested to be a simple and cost-effective way to achieve multiple benefits [1] Studies assessed the issue if multiple-micronutrient supplementation during pregnancy may have a benefit for mother and fetus. In a recently published Cochrane issue [18], including nine trials with over 15,000 patients, multiple-micronutrient supplementations, compared with micronutrients containing two or less ingredients or no supplementation/placebo, resulted in a statistically significant reduction in the number of low birth weight neonates RR 0.83; 95\% Cl 0.76-0.91, small-for-gestational-age babies (RR 0.92; 95\% Cl 0.86-0.99) and maternal anemia (RR 0.61; Cl 0.52-0.71). But the authors concluded that these results lost statistical significance when multiple-micronutrients were compared with iron folic acid supplementation. Furthermore, no statistically 
Table 2 Special groups that are at risk for undernourishment for micronutrients.

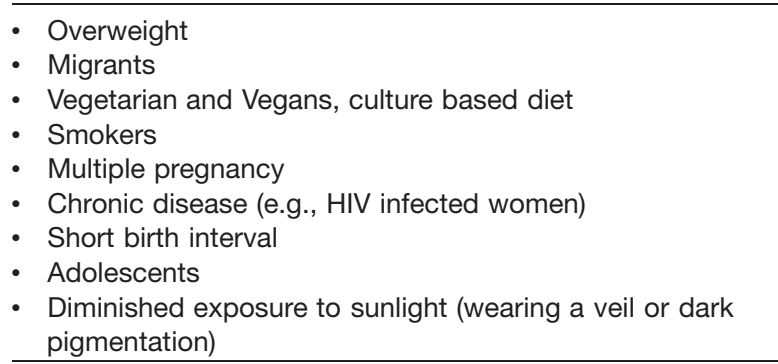

significant differences could be shown for the rates of preterm deliveries and overall perinatal mortality. However, different dose regimens were used. Table 2 includes data from women with a higher risk to be undernourished with micronutrients.

\section{Supplementation with different vitamins for preventing miscarriage}

Supplements, such as folate, have been recommended for patients at risk of spontaneous miscarriage, associated with elevated homocysteine levels. These are significantly elevated in patients with a history of two or more consecutive miscarriages [10]. As a consequence, a supplementation with folate may decrease the risk. Furthermore, oxidative stress, leading to elevated levels of reactive oxygen molecules and lowered levels of antioxidants, has been linked to spontaneous and recurrent miscarriage [24]. However, the use of supplements in pregnancy needs to be supervised, especially regarding safety of the ingredients. This fact is particularly important for the intake during the first trimester, where there exists an increased potential for teratogenicity. A teratogenic potential is noted for high maternal levels of preformed vitamin A (retinoic acid), resulting in an increased risk of spontaneous miscarriages and fetal cerebral and cardiac malformations. In contrast, a teratogenic effect of other vitamins has not been reported. Rumbold et al. [32] reported the possible benefit of vitamin supplementation for preventing miscarriage. The authors combined data of 35,812 women. No significant difference was observed between women taking any vitamins compared with controls for total fetal loss (RR 1.05, 95\% $\mathrm{Cl} 0.95-1.15)$, early or late miscarriage (RR 1.08, 95\% Cl 0.95-1.24) or stillbirth (RR $0.85,95 \% \mathrm{Cl} 0.63-1.14$ ). In four trials with 5580 patients, the authors observed that patients taking any type of vitamin(s) were less likely to develop preeclampsia (RR $0.68,95 \% \mathrm{Cl} 0.54-0.85)$ and were more likely to have a multiple pregnancy (RR 1.38, $95 \% \mathrm{Cl} 1.12-1.70)$ compared with controls. The authors conclude that vitamin supplements taken periconceptionally and in the first trimester, do not significantly reduce the risk for miscarriage or stillbirth. However, the intake of vitamin supplements may be associated with a lower rate of preeclampsia and a higher rate of multiple pregnancies.

\section{Multivitamins or folic acid for the primary prevention of birth defects}

The periconceptional use of folic acid alone or in combination with other supplements is effective for the primary prevention of neural-tube defect. A risk reduction of the occurrence and recurrence rate of neural tube defects (NTDs) has been demonstrated [4]. Furthermore, multivitamins, as well as folic acid alone, can prevent some congenital cardiovascular malformations. It becomes apparent that many women do not consume enough folic acid. The National Diet and Nutrition Survey showed that $84 \%$ of women fail to achieve the Reference Nutritient Intake for folic acid [21]. However, this statement cannot be assessed without taking the sociological and cultural background into account.

One study assessed the question if multivitamins containing $0.4-0.8 \mathrm{mg}$ of folic acid were more effective for the reduction of neural-tube defects than higher doses of folic acid, including pooled data of two Hungarian trials using a multivitamin containing $0.8 \mathrm{mg}$ folic acid and the data of the Hungarian Case-Control Surveillance of Congenital Abnormalities using a high dose of folic acid [8]. The study demonstrated that multivitamin preparations containing $0.4-0.8 \mathrm{mg}$ of folic acid were more effective for the reduction of NTDs than a high dose of folic acid. Similarly, only multivitamins were able to reduce the prevalence of congenital obstructive urinary malformations, limb deficiencies and congenital pyloric stenosis. However, folic acid was effective in reducing the rate of rectal/ anal stenosis/atresia, and a high dose of folic acid reduces the rate of orofacial clefts. These findings are in line with other studies demonstrating that the periconceptional multivitamin and folic acid supplementation reduces the overall occurrence of congenital abnormalities in addition to the previous demonstrated effect on NTDs [9].

\section{Supplementation with vitamins and preeclampsia}

A double-blind trial of 283 pregnant women with a previous preeclampsia or abnormal blood flow to the placenta assessed the effect of vitamin E $(400 \mathrm{lU} / \mathrm{d})$ plus $1000 \mathrm{mg}$ vitamin $\mathrm{C}$ versus placebo on plasma markers of vascular endothelial activation and placental insufficiency and the occurrence of preeclampsia. The study showed a possible benefit in the prevention of preeclampsia in women at increased risk of the disease [7]. These promising data are in contrast to two recently published multicenter trials. Rumbold et al. [33] included 1877 nulliparous women between 14 and 22 weeks' gestation. A total of 935 patients were randomly assigned to the vitamin group with $1000 \mathrm{mg}$ of vitamin C and $400 \mathrm{IU}$ of vitamin $\mathrm{E}$. The study showed no significant differences 
between the vitamin and placebo groups regarding the risk of preeclampsia $(6.0 \%$ and $5.0 \%$, respectively; RR $1.20 ; 95 \% \mathrm{Cl} 0.82-1.75)$, but a trend towards a higher rate of death or serious outcomes in the infant $(9.5 \%$ and 12.1\%; RR 0.79; 95\% Cl 0.61-1.02), or towards having an infant with a birth weight below the 10th percentile (8.7\% and 9.9\%; RR 0.87; 95\% Cl 0.66-1.16) in the vitamin group. The authors concluded that a supplementation with vitamins $C$ and $E$ during pregnancy does not reduce the risk of preeclampsia in nulliparous women. However, the accompanying editorial stated that this trial was powered to detect a reduction of $50 \%$ or more in the risk of preeclampsia and that the possibility of a smaller benefit could not be ruled out.

A recently published trial with 2400 high-risk pregnant patients did not show a reduced incidence after treatment with vitamin C (1000 mg) and vitamin E (400 IU) in a high risk population [31]. The results may be biased by the inhomogeneity of the study population with singleton and multiple pregnancies, patients with diabetes, chronic hypertension or antiphospholipid syndrome.

Another recent study, including 1835 pregnant women enrolled in the Pregnancy Exposures and Preeclampsia Prevention Study, showed that a regular use of multivitamins is associated with a $45 \%$ reduction of preeclampsia compared with non use (OR $0.55,95 \% \mathrm{Cl} 0.32-0.95)$ [5]. However, overweight before conception modified this effect. After adjustment for this variable, lean multivitamin users $(\mathrm{BMI}<25)$ had a $71 \%$ reduction in preeclampsia risk compared with lean nonusers (OR $0.29,95 \% \mathrm{Cl}$ $0.12-0.65)$. In contrast, there was no relation between multivitamin use and preeclampsia among overweight women $(\mathrm{BMI}>25)(\mathrm{OR} 1.08,95 \% \mathrm{Cl} 0.52-2.25)$.

\section{Iron deficiency}

At least 50 million pregnant women in low-income countries are anemic, primarily due to iron deficiency [38]. Iron-deficient anemia, the late manifestation of chronic iron deficiency, is thought to be the most common nutrient deficiency among pregnant women [43]. Anemia in pregnancy is diagnosed if hemoglobin $(\mathrm{Hb})$ concentration is lower than $110 \mathrm{~g} / \mathrm{L}$ during the first and third trimesters or lower than $105 \mathrm{~g} / \mathrm{L}$ during the second trimester, according to recommended U.S. Centers for Disease Control and Prevention (CDC) and World Health Organization (WHO) cut-off points.

Forty trials, involving 12,706 women, were included in the relevant Cochrane review [30]. Iron deficiency results in anemia, which may increase the risk of death from hemorrhage after delivery, although its effects on fetal development and birth outcomes are still unclear.

The data suggest that daily antenatal iron supplementation increases hemoglobin levels in maternal blood both antenatally and postnatally. It is difficult to quantify this increase due to a significant heterogeneity between the studies. Women who receive daily antenatal iron sup- plementation are less likely to have iron deficiency and iron-deficiency anemia at term as defined by the current cut-off values. Side-effects and hemoconcentration are more common in women who receive daily iron supplementation. No differences were evident between daily and weekly supplementation with regards to gestational anemia.

\section{Conclusion}

The adequate supply with micronutrients and omega 3FA is often not fulfilled as well in the industrialized as in low income countries. Although awareness for the preconceptional use of MV and the risk reduction for fetal anomalies is high, the appropriate pre-conceptional use is low. Obstetricians should acquire more knowledge related to nutrition and information about dietary habits. Especially, intake of omega 3-FA should be considered early in the antenatal care. Regarding the micronutritient status of a pregnant woman, a personal interview and biochemical tests during antenatal care visits can reveal micronutritient deficiencies. After counseling, these should be corrected with an individual supplementation.

\section{References}

[1] Alnwick D: Weekly iodine supplements work. Am J Clin Nutr 67 (1998) 1103

[2] Antenatal care clinical guideline. National Collaborating Centre for women's and children's health. Commissioned by the National Institute for clinical excellence, October 2003, RCOG press

[3] Baerlocher K, B Brüschweiler, E Camenzind-Frey, J Diezi, I Hösli, R Huch, et al.: Ernährung in Schwangerschaft und Stillzeit. Gefahren für Mutter und Kind? Expertenbericht der Eidgenössischen Ernährungs-Kommission für eine optimale Ernährung von Mutter und Kind BAG 2007 in press

[4] Bailey L, G Rampersaud, G Kauwell: Folic acid supplements and fortification affect the risk of neural tube defects, vascular diseases and cancer: Evolving science. J Nutr 133 (2003) 1961S

[5] Bodnar LM, G Tang, RB Ness, G Harger, JM Roberts: Periconceptional multivitamin use reduces the risk of preeclampsia. Am J Epidemiol 164 (2006) 470

[6] Catalano PM, M Hoegh, J Minium, L Hustin-Presley, S Bernard, S Kalhan, et al.: The short-and long-term implications of maternal obesity on the mother and her offspring. Br J Obstet Gynecol 113 (2006) 1126

[7] Chappell LC, PT Seed, AL Briley, FJ Kelly, R Lee, BJ Hunt, et al.: Effect of antioxidants on the occurrence of preeclampsia in women at increased risk: a randomised trial. Lancet 354 (1999) 810

[8] Czeizel AE: The primary prevention of birth defects: Multivitamins or folic acid? Int J Med Sci 1 (2004) 50

[9] Daly S, A Cotter, AE Molloy, J Scott: Homocysteine and folic acid: implications for pregnancy. Semin Vasc Med 5 (2005) 190 
[10] Del Bianco A, G Maruotti, AM Fulgieri, T Celeste, L Lombardi, NA Amato, et al.: Recurrent spontaneous miscarriages and hyperhomocysteinemia. Minerva Ginecol 56 (2004) 379

[11] Dyerberg J, HO Bang: Haemostatic function and platelet polyunsaturated fatty acids in Eskimos 1979. Nutrition 11 (1995) 475; discussion 474, 476

[12] EC, Health and Consumer Protection Directorate-General. Information Note: Methylmercury in fish and fishery products. 1-3. (2004) website:http://europa.eu.int/comm/food/ food/chemicalsafety/contaminants/information_note_mercuryfish_12-05-04.pdf

[13] Ehrenberg HM, L Dierker, C Molluzzi, B Mercer: Low maternal weight, failure to thrive in pregnancy and adverse pregnancy outcome. Am J Obstet Gynecol 189 (2003) 1126

[14] FDA/EPA. Overview of the draft FDA/EPA methylmercury (MeHg) consumer advisory. (2003). website: http://www. fda.gov/oc/opacom/mehgadvisory1011.html FDA/EPA

[15] Freeman MP: Omega-3 fatty acids and perinatal depression: a review of the literature and recommendations for future research. Prostaglandins Leukot Essent Fatty Acids Epub 75 (2006) 291

[16] Geleijnse JM, EJ Giltay, DE Grobbee, AR Donders, FJ Kok: Blood pressure response to fish oil supplementation: metaregression analysis of randomized trials. J Hypertens 20 (2002) 1493

[17] Gerrard J, D Popeski, L Ebbeling, P Brown, G Hornstra: Dietary omega 3 fatty acids and gestational hypertension in the Inuit. Arctic Med Res Suppl (1991) 763

[18] Haider BA, ZA Bhutta: Multiple-micronutrient supplementation for women during pregnancy. Cochrane Database Syst Rev 4 (2006) CD004905. DOI: 10.1002/14651858. CD004905.pub2

[19] Hibbeln JR: Seafood consumption, the DHA content of mothers' milk and prevalence rates of postpartum depression: a cross-national, ecological analysis. J Affect Disord 69 (20029) 15

[20] Hibbeln JR, JM Davis, J Heron, J Evans, DFH Wolke, J Golding: ALSPAC Study Team. Low dietary omega-3s and increased depression risk in 14541 pregnancies. American Psychiatric Association Annual Meeting 2003. San Francisco, CA. New research abstracts [Abstract Nr 4.18]

[21] Hoare J, L Henderson, CJ Bates: The National Diet and Nutrition Survey: adults aged 19-64 years. Volume 5: summary report. HMSO, London, 2005

[22] Hornstra G, R Uauy, X Yang: The impact of maternal nutrition on the offspring. Nestec and S Karger AG, Basel, Switzerland, 2005

[23] Innis SM, SL Elias: Intakes of essential n-6 and n-3 polyunsaturated fatty acids among pregnant Canadian women. Am J Clin Nutr 77 (2003) 473

[24] Jauniaux E, AL Watson, J Hempstock, YP Bao, JN Skepper, GJ Nurton: Onset of maternal arterial blood flow and placental oxidative stress: a possible factor in human early pregnancy failure. Am J Pathol 157 (2000) 2111

[25] Llorente AM, CL Jensen, RG Voigt, JK Fraley, MC Berretta, WC Heird: Effect of maternal docosahexaenoic acid supplementation on postpartum depression and information processing. Am J Obstet Gynecol 188 (2003)1348

[26] Makrides M, L Duley, SF Olsen: Marine oil, and other prostaglandin precursor, supplementation for pregnancy uncomplicated by pre-eclampsia or intrauterine growth restriction. Cochrane Database Syst Rev 19 (2006) CD003402

[27] Obesity: weighty matters website: www.gsk.com/infocus/ obesity.htm

[28] Oken E, KP Kleinman, WE Berland, SR Simon, JW RichEdwards, MW Gillman: Decline in fish consumption among pregnant women after a national mercury advisory. Obstet Gynecol 102 (2003) 346

[29] Olsen SF, P Grandjean, P Weihe, T Videro: Frequency of seafood intake in pregnancy as a determinant of birth weight: evidence for a dose dependent relationship. J Epidemiol Community Health 47 (1993) 436

[30] Pena-Rosas JP, FE Viteri: Effects of routine oral iron supplementation with or without folic acid for women during pregnancy. Cochrane Database Syst Rev 3 (2006) CD004736. DOI: 10.1002/14651858.CD004736.pub2

[31] Poston L, AL Briley, PT Seed, FJ Kelly, AH Shennan: Vitamins in Preeclampsia (VIP) Trial Consortium. Vitamin C and vitamin $\mathrm{E}$ in pregnant women at risk for pre-eclampsia (VIP trial): randomised placebo-controlled trial. Lancet 367 (2006) 1145

[32] Rumbold AR, P Middleton, CA Crowther: Vitamin supplementation for preventing miscarriage. Cochrane Database Syst Rev 2 (2005) CD004073. DOI: 10.1002/14651858. CD004073.pub2

[33] Rumbold AR, CA Crowther, RR Haslam, GA Dekker, JS Robinson: ACTS Study Group. Vitamins $C$ and $E$ and the risks of preeclampsia and perinatal complications. N Engl J Med 354 (2006) 1796

[34] Scientific Advisory Committee on Nutrition (SACN). Advice on fish consumption: benefits and risks (2004) website: http://www.sacn.gov.uk/reports

[35] Shoji H, C Franke, C Campoy, M Rivero, H Demmelmair B Koletzko: Effect of docosahexaenoic acid and eicosapentaenoic acid supplementation on oxidative stress levels during pregnancy. Free Radic Res 40 (2006) 379

[36] Simopoulos AP: The importance of the ratio of omega-6/ omega- 3 essential fatty acids. Biomed Pharmacother 56 (2002) 365

[37] Sontrop J, MK Campbell: Omega-3 polyunsaturated fatty acids and depression: a review of the evidence and a methodological critique. Prev Med 42 (2006) 4-13. Epub 2005 Dec 7

[38] Stoltzfus RJ. Iron deficiency and strategies for its control. Report prepared for the Office of Nutrition. USAID 1995

[39] Szajewska H, A Horvath, B Koletzko: Effect of n-3 longchain polyunsaturated fatty acid supplementation of women with low-risk pregnancies on pregnancy outcomes and growth measures at birth: a meta-analysis of randomized controlled trials. Am J Clin Nutr 83 (2006) 1337

[40] Tanskanen A, JR Hibbeln, J Hintikka, K Haatainen, K Honkalampi, H Viinamaki: Fish consumption, depression, and suicidality in a general population. Arch Gen Psychiatry 58 (2001) 512

[41] What you need to know about mercury in fish and shellfish (2004). website: http://vm.cfsan.fda.gov/ dms/admehg3. $\mathrm{html})$

[42] Williams MA, et al.: Omega 3 fatty acids in maternal erythrocytes and risk for preeclampsia. Epidemiology 3 (1995) 232

[43] World Health Organization. The prevalence of anaemia in women: a tabulation of available information. 2nd Edition. World Health Organization, Geneva, 1992

[44] website: www.perilip.org 